\author{
Carlo Ricotta $\cdot$ Piermaria Corona $\cdot$ Marco Marchetti \\ Gherardo Chirici
}

\title{
On parametric fragmentation measures
}

Received: 5 May 2005/ Accepted: 7 April 2006

(C) Springer-Verlag 2006

\begin{abstract}
In the landscape ecological literature, a number of measures have been proposed for quantifying landscape fragmentation based on distinct objectives and motivations. However, none seems to be generally preferred. The main reason for this disagreement is that, from a statistical viewpoint, by mapping fragmentation into a single scalar, information is necessarily lost and no ideal function is able to uniquely characterize all aspects of landscape fragmentation. A more complete summarization of fragmentation is possible if, instead of one single index, a parametric index family is applied whose members have varying sensitivities to the presence of large and small landscape patches. While traditional indices supply point descriptions of fragmentation, according to a parametric fragmentation family $H_{\alpha}$, there is a continuum of possible fragmentation measures that differ in their sensitivity to the presence of large and small patches as a function of the scaling parameter $\alpha$. Therefore, changing $\alpha$ allows for vector description of fragmentation. The purpose of this paper is to introduce a parametric generalization of Shannon's entropy to summarize landscape fragmentation. A small set of artificial landscapes is used to clarify our proposal.
\end{abstract}

Communicated by Michel Köhl

C. Ricotta $(\bowtie)$

Department of Plant Biology, University of Rome "La Sapienza", Piazzale Aldo Moro, 00185 Rome, Italy

E-mail: carlo.ricotta@uniroma1.it

Tel.: + 39-06-49912408

Fax: + 39-06-4457540

P. Corona

Department of Forest Resources and Environment, University of Tuscia, Via San Camillo de Lellis, 01100 Viterbo, Italy

M. Marchetti · G. Chirici

Department of Environmental Sciences, University of Molise, Via Mazzini 8, 86170 Isernia, Italy
Keywords Fragmentation profile - Information theory $\cdot$ Parametric entropy

\section{Introduction}

The increased concern for environmental management has stimulated the development of multiresource forest inventories that combine different data sources such as field assessments and remote sensing to enable a global view of landscape mosaics. In this view, we can refer to landscape ecology as a methodological frame (e.g., Kleinn and Traub 2003).

Landscape ecology deals with the distribution of ecosystems in space and hypothesizes that the spatial arrangement of ecosystems has ecological implications (Turner 1990). However, before the interaction between landscape structure and ecological processes can be understood, landscape structure must be identified and quantified in (ecologically) meaningful ways. Recent overviews about harmonized/standardized forest inventory systems reiterate this point (e.g., Lund 1998; Corona and Marchetti 2000; Köhl 2003).

Landscape fragmentation is defined as "the breaking up of habitats, ecosystems or land use types into smaller parcels" (Forman 1995). Fragmentation occurs in natural systems through natural disturbances, i.e., fire, windstorms, irruptive insects and pathogens (Pickett and White 1985; Weber and Flannigan 1997). Yet, the most important cause of habitat fragmentation is the expansion and intensification of human land uses (Friedenburg 1997; Olff and Ritchie 2002).

Recent studies indicate that fragmentation has several environmental and ecological consequences. For example, it affects metapopulation dynamics (Hanski 1998; Hanski and Ovaskainen 2000) and community composition (Laurance et al. 1998; Crooks and Soulé 1999). Fragmentation also causes substantial increase in population extinctions (Gonzalez et al. 1998; Burkey 1999).

A vast variety of measures have been proposed for quantifying the fragmentation of land cover maps as a 